UC-34d

Issued: November 1982

LAT- $-9556-11 \mathrm{~S}$

1203004095

\title{
Consideration of a Measurement of $\left|\eta_{00}\right|^{2} /\left|\eta_{+-}\right|^{2}$ at LAMPF II
}

James W. Cronin*

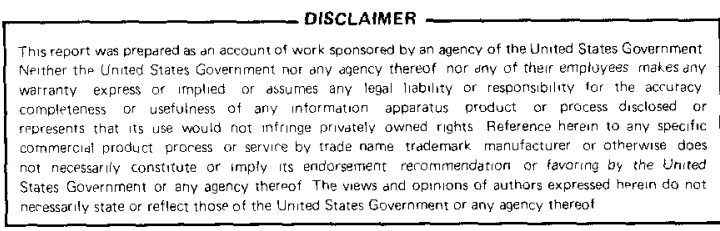

*VIsitıng Staff Member at Los Alamos. Enrico Fermi Institute, University of Chicago, 5630 South Alice, Chicago, IL 60637.
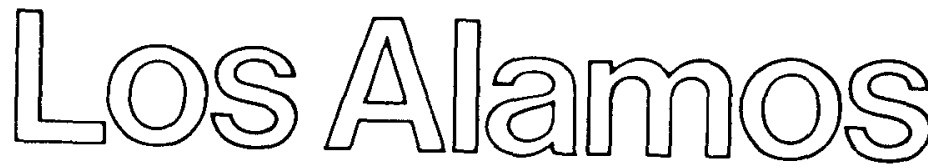

Los Alamos National Laboratory Los Alamos, New Mexico 87545

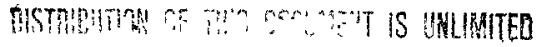




\section{DISCLAIMER}

This report was prepared as an account of work sponsored by an agency of the United States Government. Neither the United States Government nor any agency Thereof, nor any of their employees, makes any warranty, express or implied, or assumes any legal liability or responsibility for the accuracy, completeness, or usefulness of any information, apparatus, product, or process disclosed, or represents that its use would not infringe privately owned rights. Reference herein to any specific commercial product, process, or service by trade name, trademark, manufacturer, or otherwise does not necessarily constitute or imply its endorsement, recommendation, or favoring by the United States Government or any agency thereof. The views and opinions of authors expressed herein do not necessarily state or reflect those of the United States Government or any agency thereof. 


\section{DISCLAIMER}

Portions of this document may be illegible in electronic image products. Images are produced from the best available original document. 


$$
\text { CONSIDERATION OF A MEASUREMENT OF }\left.\ln _{00}\right|^{2} /\left|\eta_{+}\right|^{2}
$$

AT LAMPF II

by

James W. Cronin

\begin{abstract}
The possibility of measuring $\left|n_{00}\right|^{2} /\left|\eta_{+}\right|^{2}$ with an accuracy of better than $0.5 \%$ at LAMPF II is explored. A possible design of an experiment is presented.
\end{abstract}

This study, although incomplete, indicates that a measurement of $\left|n_{00}\right|^{2} /\left|n_{+}\right|^{2}$ could be done very well using a high-intensity accelerator of $16-$ to $18-\mathrm{GeV}$ primary proton energy. In our study, which is based on considerable experience with this measurement, we seek an error on $\left|n_{00}\right|^{2} /\left|n_{+}\right|^{2}$ of $0.5 \%$ or less. The idea of the experiment is similar to a recent one carried out at Fermi National Laboratory (Fermilab) using an 804-block lead-glass detector.

In separate runs, we compare $K_{L} \rightarrow \pi^{0} \pi^{0} / K_{S}+\pi^{0} \pi^{0}$ and $K_{L} \rightarrow \pi^{+} \pi / K_{S}+\pi^{+} \pi^{-}$. In the case of the first ratio, two $\mathrm{K}_{\mathrm{L}}$ beams, each $1 \mathrm{~cm}^{2}$, are prepared side by side, $30 \mathrm{~cm}$ apart. A regenerator alternates between the beams. The detector, which consists of $1024 \mathrm{NaI}$ blocks, each $60 \mathrm{~mm}$ by $60 \mathrm{~mm}$ by 14 radiation lengths deep, is assumed to have an energy resolution of $5 \%$ (std dev) and a position resolution for photons of $1.0 \mathrm{~cm}$ ( $\mathrm{std} \mathrm{dev}$ ). The mean momentum of the accepted $\mathrm{K}_{\mathrm{L}}$ decays is $4 \mathrm{GeV} / \mathrm{c}$. 
The beams are produced by $18.8-\mathrm{GeV}$ protons with an interaction rate of $4 \times 10^{12} / \mathrm{s}$ on a suitable target. A distance of $16 \mathrm{~m}$ is used to collimate the beam to produce two $1-$ by $1-\mathrm{cm}$ beams separated by $30 \mathrm{~cm}$ at $20 \mathrm{~m}$ from the target. The $20-\mathrm{m}$ distance is chosen so that as much as $3.25 \mathrm{~m}$ of carbon can be used as a neutron absorber, with good geometry. We have considered the production of $\mathrm{K}^{0}$ by $\mathrm{K}^{+}$beams and $\pi$ beams, but the yields are very inferior for this experiment.

Figure 1 shows a sketch of the apparatus. The regenerator is moved back and forth each second, which may cause some loss of time at a dc machine. The presence of the regenerator and free-decay sources simultaneously in front of the detector eliminates a variety of monitoring problems and, more important, keeps the singles rates constant for the $\mathrm{K}_{\mathrm{S}}-\mathrm{K}_{\mathrm{L}}$ comparison.

The price paid for this nice feature is that we must have a technique to identify the beam from which a given event has come. The detector measures only positions and energies of photons. For a four-photon event $\left(a+2 \pi^{0}\right.$ candidate), we can locate the distance from the apparatus for which $\mathrm{K}_{\mathrm{L}}+2 \pi^{0}$ provides a best fit. Experience shows that we can find this position with a precision of about $0.25-\mathrm{K}_{\mathrm{S}}$ lifetimes. (Finding a good solution at this point already suppresses $\mathrm{K}_{\mathrm{L}} \rightarrow 3 \pi^{0}$ where $2 \gamma$ are 1ost. We will not discuss in detail the rejection of $3 \pi^{0}$ because we did not have time to investigate this point carefully. Experience with our Fermilab apparatus suggests that the $3 \pi^{0}$ background would not be too large.)

We propose here a new technique to identify the beams and to measure the transverse momentum of the regenerated $\mathrm{K}_{\mathrm{S}}$ decays. We select beams comparable to or smaller in size than the error in the center-of-energy determination of the NaI array. We also separate the two beams so that the transverse momentum required of an event from one beam to place its center of energy on the other beam is larger than the characteristic transverse momentum of the incoherent regeneration. In fact, we make it comparable to the characteristic inelastic transverse momentum.

Specifically, for the proposed apparatus the mean distance from the decay point to the $\mathrm{NaI}$ is $3 \mathrm{~m}$, and the beam separation is $0.3 \mathrm{~m}$. With a mean momentum of $4 \mathrm{GeV} / \mathrm{c}$, the transverse momentum separation is $400 \mathrm{MeV} / \mathrm{c}$. The distribution of incoherent regeneration is proportional to $\exp \left[\left(\mathrm{P}_{\perp} / 110\right)^{2}\right]$, and the inelastic is proportional to $\left.\exp \left[\mathrm{P}_{\perp} / 350\right)^{2}\right]$. Thus, beam crosstalk from incoherent regeneration is clearly negligible. 
An important parameter is the precision with which the center of energy is determined. This precision is a convolution of the beam size and the errors on the center of energy from the finite energy and position resolutions of the detector. For the case studied here, the $x$ and $y$ components of the center of energy have an $\mathrm{rms}$ value of $\sim 1 \mathrm{~cm}$. The rms spread of the inelastic distribution is $[(350 \cdot 300) / 4000]=27 \mathrm{~cm}$. Thus, the crosstalk from inelastic events is $\sim(1 / 27)^{2} \times \mathrm{R} \times \exp (30 / 27)^{2}$, where $\mathrm{R}$ is the ratio of inelastic events in the regenerator beam to free-decay events in the other beam. Typically, we run so that the coherent regenerated event rate is $\sim 5$ times that for free decay. At the lower energies involved, the inelastic events are typically about the same size as the coherent events, so the crosstalk is $\sim 5 \times(1 / 27)^{2} \times \mathrm{e}^{-1}=0.2 \%$. This amount is easily corrected for.

A more important consideration in the use of this small double-beam technique is the ability to separate the incoherently regenerated events from the coherent ones. It is the ratio of free-decay events to coherent events that we want to measure. It is necessary to subtract the incoherent events, which is done by extrapolation. The size of the subtraction is the ratio of the rms radius of the center-of-energy distribution $(1 \mathrm{~cm})$ to the rms radius of the incoherent distribution $(8.25 \mathrm{~cm})$. The subtraction is $(1 / 8.25)^{2}=1.5 \%$. Experience shows that this subtraction can be made to better than $10 \%$ of its size, so that the uncertainty in this subtraction is $\sim 0.15 \%$. Expressed in more physical terms, once the beam for a given $K+\pi^{0} \pi^{0}$ event is determined, the transverse momentum of the $\mathrm{K}$ can be determined to $13 \mathrm{MeV} / \mathrm{c}$. The spread of the carbon incoherent regeneration is $110 \mathrm{MeV} / \mathrm{c}$.

Given that the small-beam technique is satisfactory, a number of advantages become apparent. The absolute efficiency of the detector is very high. As a consequence, a good yield can be obtained without a large amount of incident beam. Also, since at a high-intensity accelerator there is a large primary proton flux, we can afford to attenuate the beam with a carbon absorber to reduce the neutron contamination.

We have calculated the efficiency of the detector described in the beginning of this report and have evaluated its response to the $\mathrm{K}_{\mathrm{L}}$ beam produced at $100 \mathrm{mrad}$. Figure 2 gives the efficiency of the detector as a function of $\mathrm{K} \rightarrow \pi^{0} \pi^{0}$ decay distance and momentum. We have chosen $2 \mathrm{~m}$ as the minimum distance so that no angles of photons incident on the detector are greater than $25^{\circ}$. 
In Fig. 3 we give the response of the detector to the available $\mathrm{K}$ beam. The match of the $\mathrm{K}$ spectrum to the detector is not excellent, but it is satisfactory. In Table $I$, we give the rates for $K_{L}+\pi^{0} \pi^{0}$ that can be expected; we assume that the limitation in the measurement will be governed by this number. We have not worked out the best strategy for the regenerator configuration, but presumably it would be stepped through the decay region.

With a modest total rate in the beam, we see that in a $1000-\mathrm{h}$ run we can collect $>5 \times 10^{5}$ events. This assumes, of course, many other things that we have not considered. We have to trigger the device, we have to handle the $3 \pi^{0}$ events, and we have to handle the data rates.

There are a number of favorable circumstances. Among these is the fact that the high efficiency of the apparatus, principally no hole, means that only a sma11 fraction (about 10\%) of the $3 \pi^{0}$ decays gives four photons in the apparatus. Many decays will occur with very low energy, but these can be eliminated with an energy threshold on the detector. There are two distinct advantages of the low energy. One is that the inelastic $\mathrm{K}$ decays are small, about equal to the coherent events. (They are about 7 times larger at Fermilab energies.) Second, a muon will deposit about $200 \mathrm{MeV}$ in the NaI crystals, which is only a factor of 5 below the mean photon energies. Muons will be plentiful in any setup and will serve as an effective rea1-time energy calibration.

For the charged-decay part of the experiment, imagine a magnet immediately behind the NaI; the NaI can be rolled out of the way and drift chambers can be moved in. It is essential that the regeneration arrangement remain identical.

\section{ACKNOWLEDGMENTS}

I want to thank C. Hoffman, R. Mischke, and R. Carlini for helpful discussions and excellent instruction on the use of the computer. 
TABLE I

YIELDS OF DETECTED $\mathrm{K}_{\mathrm{L}} \rightarrow \pi^{0} \pi^{0}$ FOR

$4 \times 10^{12}$ INTERACTING $18.8-G e V / C$ PROTONS

Carbon Absorber

(m)

0

2.5

3.25

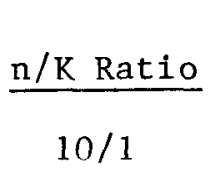

$2 / 1$

$1.2 / 1$

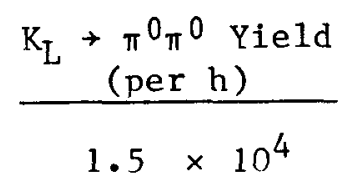

$1.5 \times 10^{3}$

$0.75 \times 10^{3}$
Total Incident Flux (pers)

$1 \times 10^{7}$

$3 \times 10^{5}$

$1 \times 10^{5}$

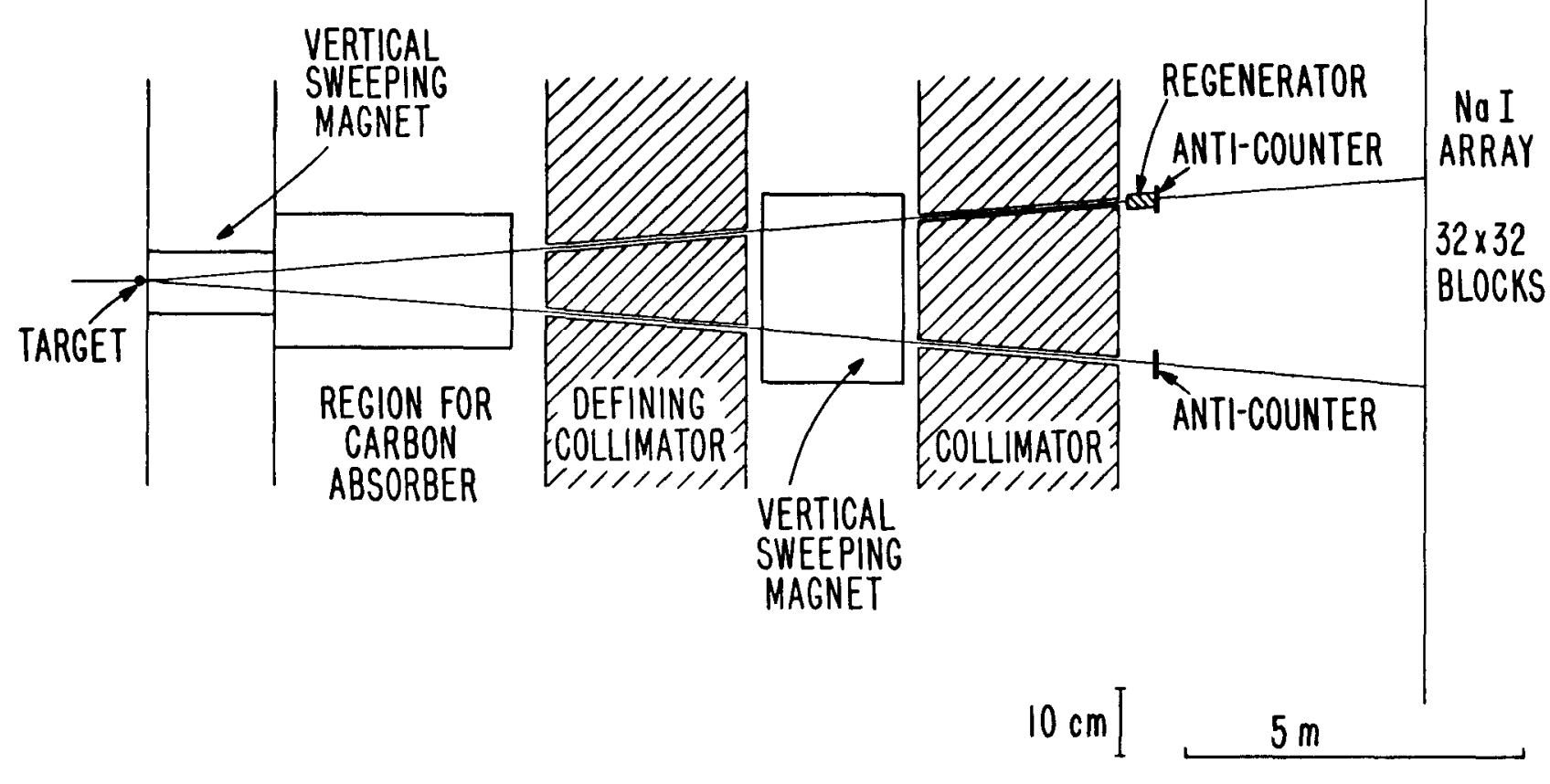

Fig. 1. Schematic of beam line and experimental apparatus. 

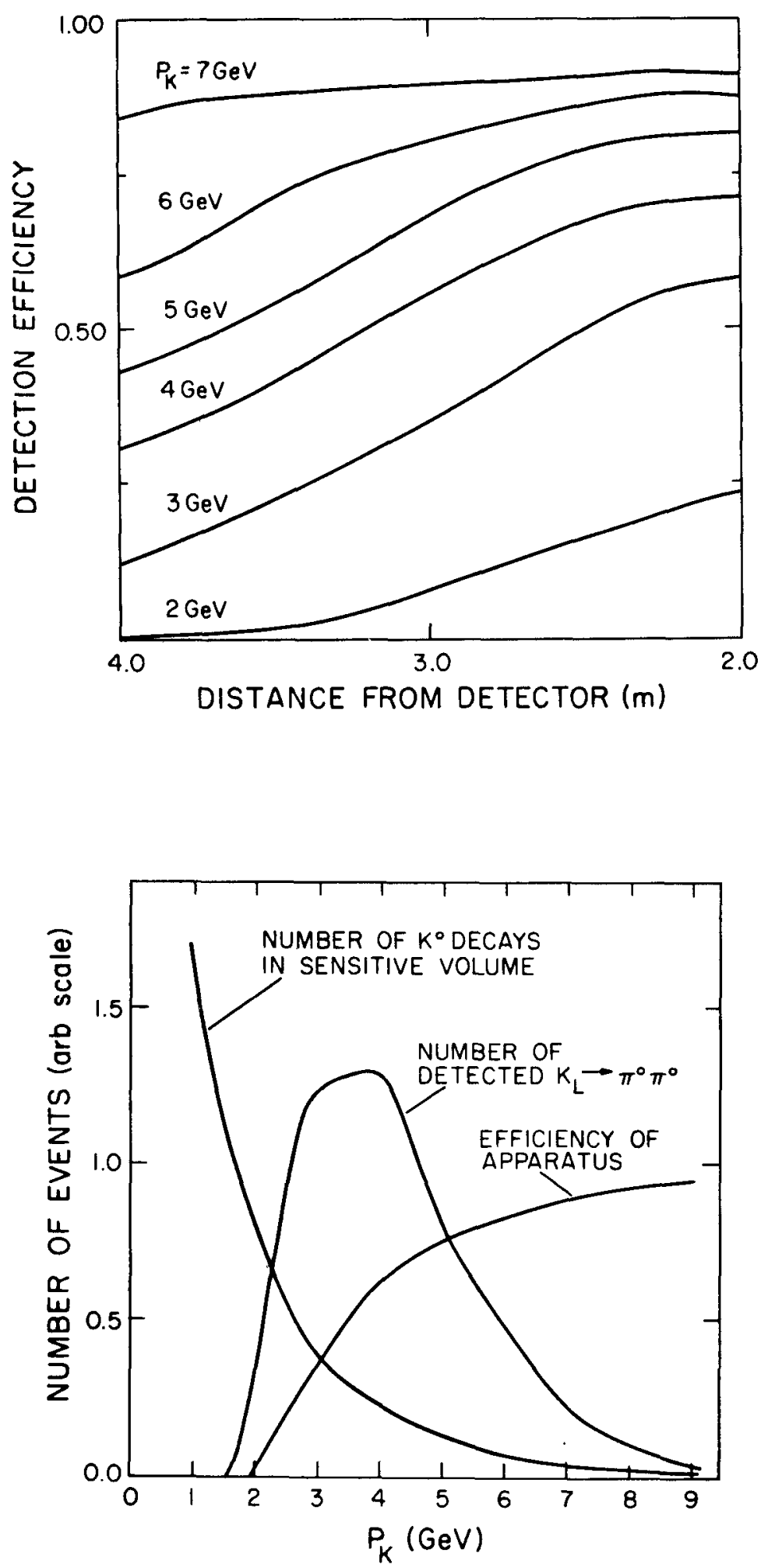

Fig. 2 .

The detection efficiency as a function of the distance from the $\mathrm{K}^{0}$ decay point to the detector, for various values of the $\mathrm{K}_{\mathrm{L}}{ }^{0}$ momentum.
Fig. 3.

The response of the apparatus to $\mathrm{K}^{0} \rightarrow \pi^{0} \pi^{0}$ as a function of kăon momentum. 